\title{
FUZZY CONGRUENCES ON GROUPS AND RINGS
}

MAROUF A. SAMHAN and T.M.G. AHSANULLAH

\author{
Department of Mathematics, King Saud University \\ P.O. Box 2455, Riyadh 11451 \\ Saudi Arabia
}

(Received December 22, 1992 and in revised form October 8, 1993)

\begin{abstract}
We prove that the lattice of fuzzy congruences of a group $G$ (resp. $\operatorname{ring} R$ ) is isomorphic to the lattice of fuzzy normal subgroups of $G$ (resp. fuzzy ideals of $R$ ).
\end{abstract}

KEY WORDS AND PHRASES. Lattice, groups, rings, fuzzy congruences, fuzzy normal subgroup, fuzzy ideals, isomorphism.

1991 AMS SUBJECT CLASSIFICATION CODES.

\section{INTRODUCTION.}

In [2], Yuan Bo and Wu Wangming investigated the relationship between the fuzzy ideals and the fuzzy congruences on a distributive lattice, and obtained that the lattice of fuzzy ideals is isomorphic to the lattice of fuzzy congruences on a generalized Boolean algebra. Our aim in this paper is to investigate this isomorphism in the varieties of groups and rings. We show that the lattice of fuzzy normal subgroups (resp. fuzzy ideals) and the lattice of fuzzy congruences on a group $G$ (resp. ring $R$ ) are isomorphic.

In doing so, we take into account the notions in [2], [3], and [4]. For the sake of completeness we recall some necessary definitions from [2], [3], and [4].

\section{FUZZY NORMAL SUBGROUPS, FUZZY IDEALS AND FUZZY CONGRUENCES.}

Throughout this paper, $G$ stands for the group $(G, *)$ with an identity element $e$, and $R$ stands for the ring $(R,+, \cdot)$. By fuzzy sets we mean maps from a set $X$ into $\langle[0,1], V, \Lambda\rangle$, where $[0,1]$ is the unit interval, $x V y=\max (x, y)$, and $x \Lambda y=\min (x, y)$.

DEFINITION 2.1. A fuzzy relation $\theta$ on a set $X$ is a fuzzy equivalence relation on $X$ if the following conditions are satisfied for all $x, y, z$, in $X$ :

(E1): $\theta(x, x)=1$;

(E2): $\theta(x, y)=\theta(y, x)$;

(E3): $\theta(x, y) \Lambda \theta(y, z) \leq \theta(x, z)$.

\section{DEFINITION 2.2 .}

(a) A fuzzy equivalence relation $\theta$ on a group $G$ is a fuzzy congruence on $G$ if the following condition is satisfied for all $x, y, z, t$ in $G$ :

(G1): $\theta(x * z, y * t) \geq \theta(x, y) \Lambda \theta(z, t)$.

(b) A fuzzy equivalence relation $\theta$ on a ring $R$ is a fuzzy congruence on $R$ if the following two conditions are satisfied for all $x, y, z, t$ in $R$ :

(R1): $\theta(x+z, y+t) \geq \theta(x, y) \Lambda \theta(z, t)$ 
(R2): $\theta(x z, y t) \geq \theta(x, y) \Lambda \theta(z, t)$.

We denote the set of all fuzzy congruences on $G$ (resp. on $R$ ) by $F C(G)$ (resp. $F C(R)$ ).

LEMMA 2.1. (a) If $\theta \in F C(G)$, and $x, y, z \in G$, then

(i) $\theta(x, y) \geq \theta(x * z, y * z) \Lambda \theta(z * x, z * y)$;

(ii) $\theta\left(x^{-1}, y^{-1}\right)=\theta(x, y)$.

(b) If $\theta \in F C(R)$, and $x, y, z \in R$, then

(i) $\theta(x, y) \geq \theta(x+z, y+z) \Lambda \theta(x z, y z) \Lambda \theta(z x, z y)$;

(ii) $\theta(-x,-y)=\theta(x, y)$.

PROOF. The proofs of the parts (a) and (b) are similar, so, we prove only part (a).

(a) (i):

$$
\begin{aligned}
\theta(x, y)=\theta\left(x * z * z^{-1}, y * z * z^{-1}\right) & \geq \theta(x * z, y * z) \Lambda \theta\left(z^{-1}, z^{-1}\right) \\
& =\theta(x * z, y * z)
\end{aligned}
$$

Similarly,

$$
\theta(x, y) \geq \theta(z * x, z * y)
$$

Therefore, combining (2.1) and (2.2) we get (i).

(ii):

$$
\begin{aligned}
\theta\left(x^{-1}, y^{-1}\right) & \geq \theta\left(x * x^{-1}, x * y^{-1}\right) \\
& =\theta\left(e, x * y^{-1}\right) \\
& \geq \theta(y, x) \\
& =\theta(x, y) .
\end{aligned}
$$

On the other hand;

$$
\theta\left(x^{-1}, y^{-1}\right) \geq \theta(x, y)
$$

implies $\theta(x, y) \geq \theta\left(x^{-1}, y^{-1}\right)$ since $x$ and $y$ are arbitrary elements.

Therefore, $\theta\left(x^{-1}, y^{-1}\right)=\theta(x, y)$.

DEFINITION 2.3. (a) If $\mu$ is a fuzzy set of $G$, then $\mu$ is a fuzzy normal subgroup of $G$ if the following conditions are satisfied for all $x, y$ in $G$ :

(N1): $\mu(x * y) \geq \mu(x) \Lambda \mu(y)$;

(N2): $\mu\left(x^{-1}\right)=\mu(x)$;

(N3): $\mu(e)=1$

(N4): $\mu(x * y)=\mu(y * x)$.

(b) If $\mu$ is a fuzzy set of $R$, then $\mu$ is a fuzzy ideal of $R$ if the following conditions are satisfied for all $x, y \in R$ :

(I1): $\mu(x+y) \geq \mu(x) \Lambda \mu(y)$

(I2): $\mu(x y) \geq \mu(x) \Lambda \mu(y)$;

(I3): $\mu(-x)=\mu(x)$;

(I4): $\mu(0)=1$;

(I5): $\mu(x y) \geq \mu(x)$ and $\mu(x y) \geq \mu(y)$.

We denote the set of all fuzzy normal subgroups of $G$ (resp. fuzzy ideals of $R$ ) by $F N(G)$ (resp. $F I(R)$ ).

It is clear that the algebraic systems

$$
<F C(G),+, \cdot>,<F I(R),+, \cdot>,
$$


$\langle F N(G),+, \cdot\rangle$, and $\langle F I(R),+, \cdot\rangle$ are lattices, where the binary operation $\cdot$ is just the intersection of fuzzy congruences (normal subgroups, ideals), and for $\mu_{1}, \mu_{2} \in F N(G)$, $\nu_{1}, \nu_{2} \in F I(R)$, and $\theta_{1}, \theta_{2} \in F C(G)$ (resp. $F C(R)$ ), the addition is defined as follows:

$$
\begin{aligned}
& \mu_{1}+\mu_{2}=\cap\left\{\mu \in F N(G): \mu \geq \mu_{1} \cup \mu_{2}\right\} \\
& \nu_{1}+\nu_{2}=\cap\left\{\nu \epsilon F I(R): \nu \geq \nu_{1} \cup \nu_{2}\right\}, \text { and } \\
& \left.\theta_{1}+\theta_{2}=\cap\{\theta \in F C(G) \text { (resp. } \theta \in F C(R)): \theta \geq \theta_{1} \cup \theta_{2}\right\} .
\end{aligned}
$$

DEFINITION 2.4. (a) Let $\mu \in F N(G)$. Define the fuzzy relation $C_{1}(\mu)$ on $G$ by $C_{1}(\mu)(x, y)=\mu\left(x * y^{-1}\right)$ for all $x, y \in G . \quad C_{1}(\mu)$ is called the fuzzy relation induced by the fuzzy normal subgroup $\mu$.

(b) Let $\nu \in F I(R)$. Define the fuzzy relation $C_{2}(\nu)$ on $R$ by $C_{2}(\nu)(x, y)=\nu(x-y)$ for all $x, y \in R . C_{2}(\nu)$ is called the fuzzy relation induced by the fuzzy ideal $\nu$ of $R$.

LEMMA 2.2. (a) If $\mu \in F N(G)$, then $C_{1}(\mu) \in F C(G)$;

(b) If $\nu \in F I(R)$, then $C_{2}(\nu) \in F C(R)$.

PROOF. (a) Put $\theta=C_{1}(\mu)$, and let $x, y, z, t \in G$.

(E1): $\theta(x, x)=\mu\left(x * x^{-1}\right)=\mu(e)=1$.

(E2): $\theta(x, y)=\mu\left(x * y^{-1}\right)=\mu\left(\left(x * y^{-1}\right)^{-1}\right)=\mu\left(y * x^{-1}\right)=\theta(y, x)$.

(E3): $\theta(x, z) \Lambda \theta(z, y)=\mu\left(x * z^{-1}\right) \Lambda \mu\left(z * y^{-1}\right)$

$$
\leq \mu\left(x * z^{-1} * z * y^{-1}\right)=\mu\left(x * y^{-1}\right)=\theta(x, y) .
$$

(G1): $\theta(x, y) \Lambda \theta(z, t)=\mu\left(x * y^{-1}\right) \Lambda \mu\left(z * t^{-1}\right)=\mu\left(y^{-1} * x\right) \Lambda \mu\left(z * t^{-1}\right)$

$$
\begin{aligned}
& \leq \mu\left(y^{-1} *\left(x * z * t^{-1}\right)\right)=\mu\left(x * z * t^{-1} * y^{-1}\right) \\
& =\mu\left((x * z) *(y * t)^{-1}\right)=\theta(x * z, y * t) .
\end{aligned}
$$

Therefore, $\theta=C_{1}(\mu) \in F C(G)$.

(b) Put $\phi=C_{2}(\nu)$, and let $x, y, z, t \in R$.

The proof of E1, E2, and E3 are similar to the proof given in part (a).

(R1): $\phi(x, z) \Lambda \phi(y, t)=\nu(x-z) \Lambda \mu(y-t)$

$$
\leq \nu((x+y)-(z+t))=\phi(x+y, z+t) .
$$

(R2): $\phi(x y, z t)=\nu(x y-z t)=\nu((x-z) y+z(y-t))$

$$
\begin{aligned}
& \geq \nu((x-z) y) \Lambda \nu(z(y-t)) . \\
& \geq \nu(x-z) \Lambda \nu(y-t)=\phi(x, z) \Lambda \phi(y, t) .
\end{aligned}
$$

Therefore, $\phi=C_{2}(\nu) \in F C(R)$.

DEFINITION 2.5. (a) Let $\theta \in F C(G)$. Define a fuzzy subset $\mu=N(\theta)$ of $G$ by $\mu(x)=\theta(e, x)$ for all $x \in G$. $N(\theta)$ is called the fuzzy subset induced by $\theta$.

(b) Let $\phi \in F C(R)$. Define a fuzzy subset $\nu=I(\phi)$ of $R$ by $\nu(x)=\phi(0, x)$ for all $x \in R$. $I(\phi)$ is called the fuzzy subset induced by $\phi$.

LEMMA 2.3. (a) If $\theta \in F C(G)$, then $N(\theta) \in F N(G)$;

(b) If $\phi \in F C(R)$, then $I(\phi) \in F I(R)$.

PROOF. (a) Put $\mu=N(\theta)$, and let $x, y, \in G$.

(N1): $\mu(x * y)=\theta(e, x * y) \geq \theta(e, x) \Lambda \theta(e, y)=\mu(x) \Lambda \mu(y)$.

(N2): $\mu\left(x^{-1}\right)=\theta\left(e, x^{-1}\right)=\theta(e, x)$

(by Lemma 2.1)

(N3): $\mu(e)=\theta(e, e)=1$.

$$
=\mu(x)
$$




$$
\text { (N4): } \begin{aligned}
\mu(x * y)=\theta(e, x * y) & \geq \theta\left(y^{-1}, x\right) \\
& \geq \theta(e, x * y) \\
& =\mu(y * x)
\end{aligned}
$$

Similarly;

$$
\mu(y * x) \geq \mu(x * y)
$$

Combining (2.3) and (2.4) we get (N4).

Therefore, $N(\theta) \in F N(G)$.

(b) Put $\nu=I(\phi)$, and let $x, y \in R$.

The proofs of (I1)-(I4) are similar to (N1)-(N3) of part (a).

(I5): $\nu(x)=\phi(0, x)=\phi(0, x) \Lambda \phi(y, y) \leq \phi(o, x y)=\nu(x y)$

Similarly,

$$
\nu(y) \leq \nu(x y)
$$

Combining (2.5) and (2.6) we get (I5).

Therefore, $I(\phi) \in F I(R)$.

THEOREM 2.1. (a) If $\mu \in F N(G)$, then $\mu=N\left(C_{1}(\mu)\right)$;

(b) If $\theta \in F C(G)$, then $\theta=C_{1}(N(\theta))$;

(c) If $\nu \in F I(R)$, then $\nu=I\left(C_{2}(\nu)\right)$;

(d) If $\phi \in F C(R)$, then $\phi=C_{2}(I(\phi))$.

PROOF. (a) Let $x$ be any element in $G$. Then $N\left(C_{1}(\mu)\right)(x)=C_{1}(\mu)(e, x)=\mu\left(e * x^{-1}\right)$ $=\mu\left(x^{-1}\right)=\mu(x)$.

(b) Let $x, y$ be any two elements in $G$. Then

On the other hand;

$$
\begin{array}{r}
C_{1}(N(\theta))(x, y)=N(\theta)\left(x * y^{-1}\right)=\theta\left(e, x * y^{-1}\right) \geq \\
=\theta(y, x) \\
=\theta(x, y)
\end{array}
$$

$$
\begin{aligned}
\theta(x, y) \geq & \theta\left(x * y^{-1}, e\right) \\
& =\theta\left(e, x * y^{-1}\right)=N(\theta)\left(x * y^{-1}\right)=C_{1}(N(\theta))(x, y)
\end{aligned}
$$

Combining (2.7) and (2.8) we get $\theta=C_{1}(N(\theta))$.

(c) Let $x$ be any element in $R$. Then

$$
I\left(C_{2}(\nu)\right)(x)=C_{2}(\nu)(0, x)=\nu(0-x)=\nu(-x)=\nu(x) .
$$

(d) Let $x, y$ be any two elements in $R$. Then

On the other hand;

$$
\begin{array}{r}
C_{2}(I(\phi))(x, y)=I(\phi)(x-y)=\phi(0, x-y) \geq \phi(y, x) \\
=\phi(x, y)
\end{array}
$$

$$
\begin{aligned}
& \phi(x, y) \geq \phi(x-y, 0) \\
&=\phi(0, x-y)=I(\phi)(x-y)=C_{2}(I(\phi))(x, y)
\end{aligned}
$$

Combining (2.9) and (2.10) we get $\phi=C_{2}(I(\phi))$.

LEMMA 2.4. Let $\mu_{1}, \mu_{2} \in F N(G)$ (resp. $\left.F I(R)\right)$, and $\theta_{1}, \theta_{2} \in F C(G)$ (resp. $\left.F C(R)\right)$. Then

(a) $\mu_{1} \subseteq \mu_{2}$ implies that $C_{1}\left(\mu_{1}\right) \subseteq C_{1}\left(\mu_{2}\right)$ (resp. $C_{2}\left(\mu_{1}\right) \subseteq C_{2}\left(\mu_{2}\right)$ ).

(b) $\theta_{1} \subseteq \theta_{2}$ implies that $N\left(\theta_{1}\right) \subseteq N\left(\theta_{2}\right)$ (resp. $I\left(\theta_{1}\right) \subseteq I\left(\theta_{2}\right)$ ). 
PROOF. Trivial.

3. THE MAIN RESULT.

PROPOSITION 3.1. (a) Let $\theta_{1}, \theta_{2} \in F C(G)$. Then

(i) $\quad N\left(\theta_{1} \cap \theta_{2}\right)=N\left(\theta_{1}\right) \cap N\left(\theta_{2}\right)$;

(ii) $N\left(\theta_{1}+\theta_{2}\right)=N\left(\theta_{1}\right)+N\left(\theta_{2}\right)$.

(b) Let $\phi_{1}, \phi_{2} \in F C(R)$. Then

(i) $\quad I\left(\phi_{1} \cap \phi_{2}\right)=I\left(\phi_{1}\right) \cap I\left(\phi_{2}\right)$

(ii) $I\left(\phi_{1}+\phi_{2}\right)=I\left(\phi_{1}\right)+I\left(\phi_{2}\right)$.

PROOF. (a) Let $x \in G$.

(i) $\quad N\left(\theta_{1} \cap \theta_{2}\right)(x)=\left(\theta_{1} \cap \theta_{2}\right)(e, x)=\theta_{1}(\epsilon, x) \cap \theta_{2}(e, x)$

$$
=N\left(\theta_{1}(x) \cap N\left(\theta_{2}\right)(x)=\left(N\left(\theta_{1}\right) \cap N\left(\theta_{2}\right)\right)(x)\right. \text {. }
$$

(ii) Since $\theta_{\imath} \subseteq \theta_{1}+\theta_{2}(i=1,2)$, it follows by Lemma 2.4 that $N\left(\theta_{\imath}\right) \subseteq N\left(\theta_{1}+\theta_{2}\right)(\imath=1,2)$. Hence, $N\left(\theta_{1}\right)+N\left(\theta_{2}\right) \subseteq N\left(\theta_{1}+\theta_{2}\right)$.

Moreover, if $\mu$ is any fuzzy normal subgroup of $G$ containing $N\left(\theta_{1}\right)$ and $N\left(\theta_{2}\right)$, then by Lemma $2.4 C_{1}\left(N\left(\theta_{1}\right)\right) \subseteq C_{1}(\mu)(i=1,2)$. But as $\theta_{1}=C_{1}\left(N\left(\theta_{1}\right)\right)(i=1,2)$, we have $\theta_{1} \subseteq C_{1}(\mu)$ $(\imath=1,2)$. Thus; $\theta_{1}+\theta_{2} \subseteq C_{1}(\mu)$. Hence, $\quad N\left(\theta_{1}+\theta_{2}\right) \subseteq N\left(C_{1}(\mu)\right)=\mu$. Therefore. $N\left(\theta_{1}+\theta_{2}\right)=N\left(\theta_{1}\right)+N\left(\theta_{2}\right)$.

(b) Similar to (a).

THEOREM 3.1. (a) $<F C(G),+, \cdot\rangle \cong\langle F N(G),+, \cdot\rangle$;

(b) $<F C(R),+, \cdot>\cong<F I(R),+, \cdot>$.

PROOF. (a) Define the function $f: F C(G) \rightarrow F N(G)$ by $f(\theta)=N(\theta)$ for $\theta \in F C(G)$.

By Proposition 3.1, $f$ is a lattice homomorphism.

To prove that is onto, let $\mu \in F N(G)$. Then in view of Lemma 2.2 and Lemma 2.3 we have $\theta=C_{1}(\mu) \in F C(G)$ such that $f(\theta)=f\left(C_{1}(\mu)\right)=N\left(C_{1}(\mu)\right)=\mu$ as desired.

To complete the proof we assume that $\theta_{1}, \theta_{2}$ in $F C(G)$ such that $f\left(\theta_{1}\right)=f\left(\theta_{2}\right)$. Then $N\left(\theta_{1}\right)=N\left(\theta_{2}\right)$, and thus, $C_{1}\left(N\left(\theta_{1}\right)\right)=C_{2}\left(N\left(\theta_{2}\right)\right)$. It follows by Theorem $2.1 \theta_{1}=\theta_{2}$, which shows that $f$ is one-to-one. Hence the result follows.

(b) Similar to (a).

\section{REFERENCES}

1. BIRKHOFF, G., Lattice Theory, AMS Colloquium Publications, Vol. XXV, 1967.

2. BO, Y. and WANGMING, W., Fuzzy ideal on a distributive lattice, Fuzzy Sets and Systems 35 (1990), 231-240.

3. LIU, W.J., Fuzzy invariant subgroups and fuzzy ideals, Fuzzy Sets and Systems 8 (1982), 133-139.

4. MUKHERJEE, N.P. and BHATTACHARYA, P., Fuzzy normal subgroups and fuzzy cosets, Information Sciences 34 (1984), 225-239.

5. ZADEH, L.A., Fuzzy Sets, Inform. and Control 8 (1965), 338-353. 


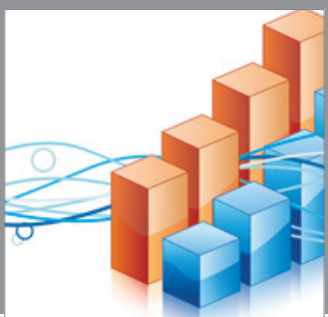

Advances in

Operations Research

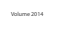

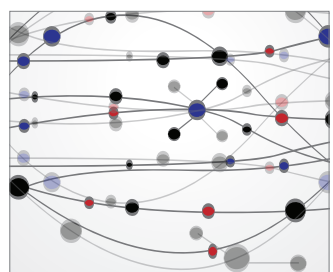

\section{The Scientific} World Journal
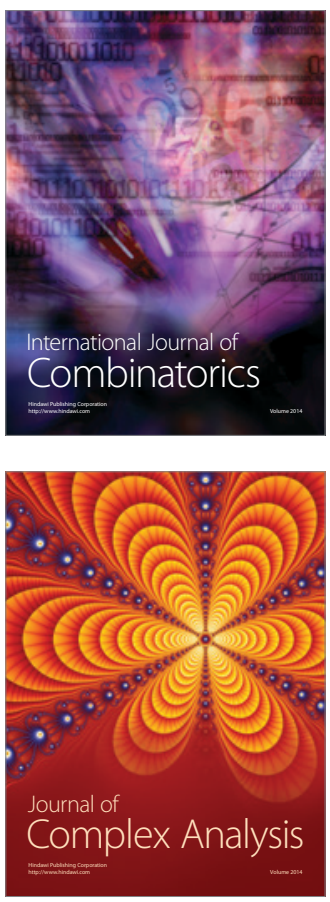

International Journal of

Mathematics and

Mathematical

Sciences
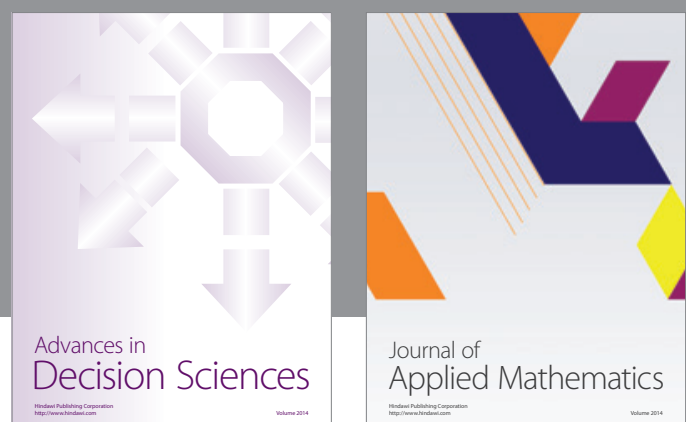

Journal of

Applied Mathematics
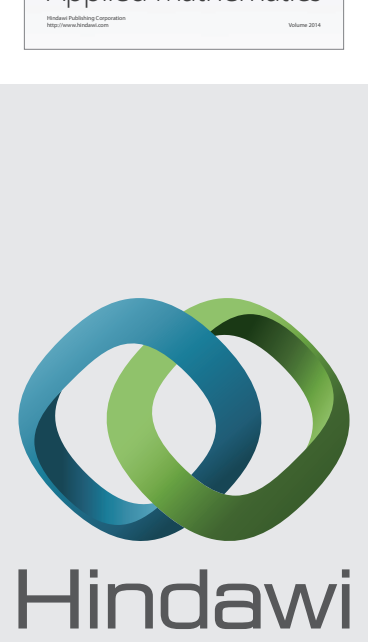

Submit your manuscripts at http://www.hindawi.com
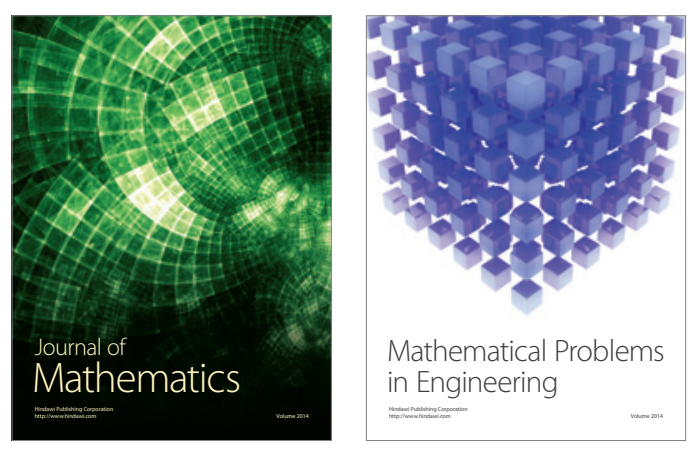

Mathematical Problems in Engineering
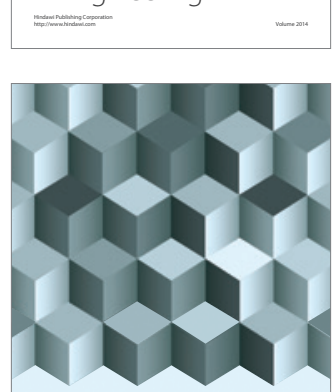

Journal of

Function Spaces
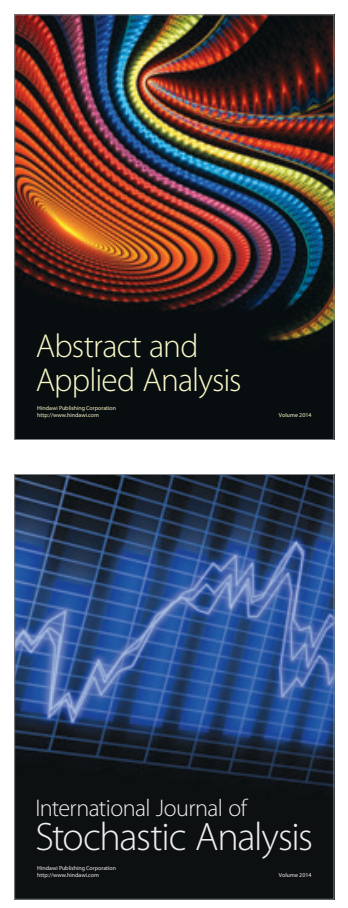

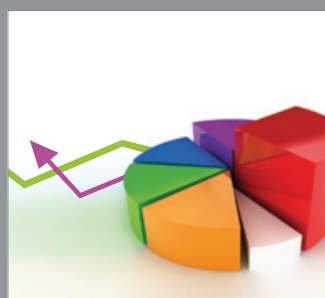

ournal of

Probability and Statistics

Promensencen
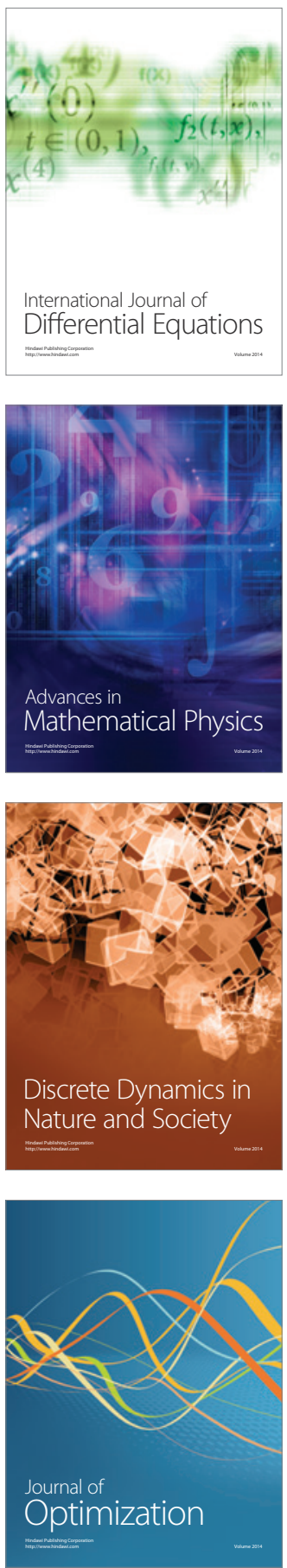